\title{
STR Profiling's Contribution to Forensic Science
}

\author{
Xunyang $\mathrm{Ye}^{1, *}$

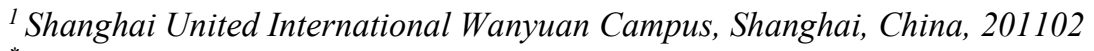 \\ ${ }^{*}$ Corresponding author. Email: Yexunyang7@gmail.com
}

\begin{abstract}
STR profiling is the edging biotechnology that uses PCR to determine the identification of an individual. Animals in the same species have more than $99 \%$ of the same DNA sequence. PCR is an analysis of the difference in the DNA. Forensic science is the usage of science in the crime scene to determine evidence and criminal procedure. Initially, scientists used to analyze the abiotic factors in the scene like furniture or left out traces that can be used to track criminals. However, using STR profiling, scientists can determine the individual with the biotic factor in the scene. Combining the outcome of the analysis with the database, the specific information of that person would be revealed. This technology dramatically improves both the efficiency and the accuracy of forensic science. People are about to get specific information and results. It helps people to accurately determine the specific person. However, it is still a new technology that is still improved. Thus, it is hoped that forensic science can still be in the next stage [3].
\end{abstract}

\section{Keywords: DNA dying, forensic science, STR profiling, PCR, fingerprint detection}

\section{INTRODUCTION}

Alec Jeffery had first discovered that in specific regions of a person's DNA. There are differences from one another, years before the murder case of Dawn Ashworth in 1986. Previously, he had been using the DNA pattern recognition machine to help solve a similar case in 1983 [1]. However, forensic science had been developed before the DNA pattern machine has invented. Thus, it is investigated how STR profiling would be able to help forensic science. Possible outcomes could be the increase in accuracy, efficiency or a change in the way people have gathered evidence. This research helps direct the way that scenic can help criminology.

\section{THE FUNCTION OF STR PROFILING AND FORENSIC SCIENCE}

Generally saying, STR profiling is the analysis of DNA which determines a person's identity with specific biological information. However, this function can be way more complex than what people have thought about in the first place. First, DNA has over 99\% similarity [9], which means that such a significant number of DNAs are the same, and only a limited region of DNA is different. The term STR means those minor differences. Furthermore, DNA is divided into two regions: intron and exon. STR occupies $10 \%-15 \%$ of the exon, which is relatively small in number. Many of these DNA pieces are located in an intron, which does not produce protein or carry genetic information [12]. Introns are removed when RNA is translated. Thus, collecting this information is extremely difficult. For completing profiling, DNA must first be isolated from the cell in order for people to analyze it. One method for isolating is by dying DNA since DNA can become visible under some colors. By dying DNA, people can get quantitative data, and scientists can see qualitative data since the structure and composition of DNA. In the article "New dye-labeled terminators for improved DNA sequencing patterns," the author has used two different dye sets to label DNA and view its pattern. It requires rhodamine dyes which cause the G-peak to be visible since it is relatively weak and occasionally $\mathrm{C}$-peak. As shown in Figure 1, G must pair with C, and A must pair with $\mathrm{T}$ since the number of each bond is different, which could not pair up if it is not set in the correct location. This method is convenient since only one reaction is enough for each template, and it does not require a primer to start. 


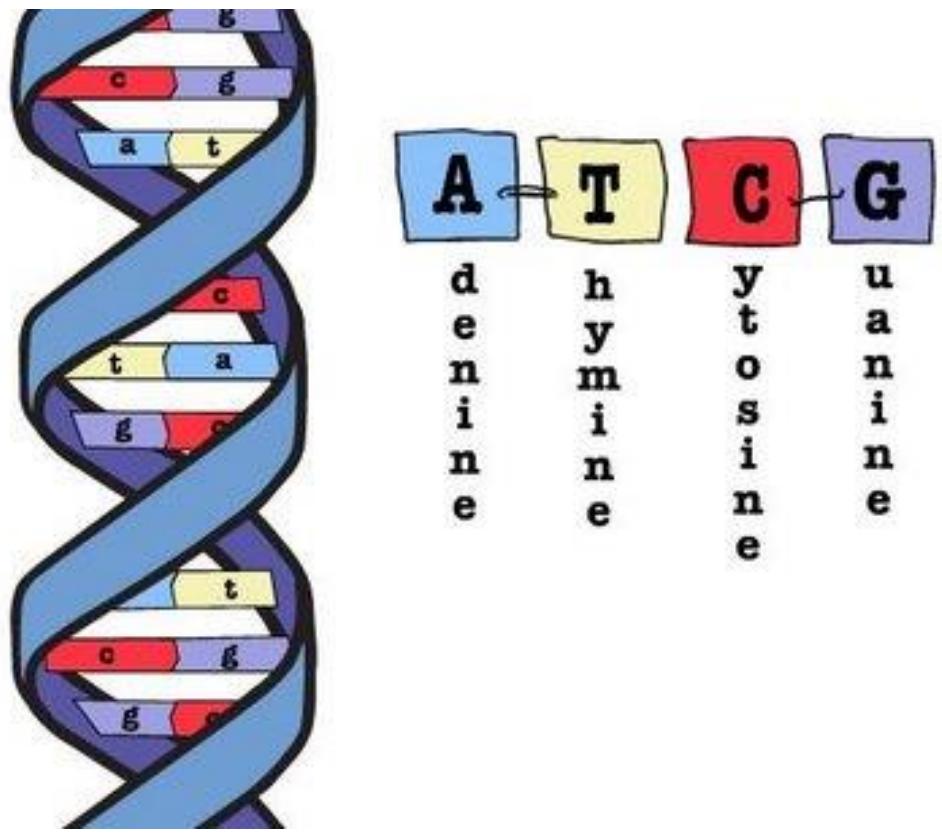

Figure 1 "The Function of Genes " [11]

Furthermore, for the most part, it can provide reliable results, which is the most crucial factor since scientists need accurate data for analysis. This first procedure has various ways to be done, and many of these STR profiling services would offer this kind of service. The second procedure is to amplify STR since they are too small for people to observe, and people need as many samples as possible for gaining an accurate outcome. This is called PCR amplification [12]. According to the book "Principles and Technical Aspects of PCR Amplification," the first procedure is to heat the DNA at about 94 degrees Celsius. DNA is a twisted, double bond shape that has two templates linked by a hydrogen bond. However, these bonds no longer hold to each other under high temperatures, and thus each template is now separated [7]. Then, the temperature starts to drop to between 40 and 65 degrees Celsius. This is because DNA polymerase needs to attach to these templates and form a new template that matches the previous template. DNA polymerase is a protein that does not function if its shape has been changed, and proteins would denature under high temperatures. Thus, the temperature must be lower in order for DNA polymerase to be functional. Once the polymerase is attached, the template can be produced. This procedure would be repeated several times to produce a large amount of that DNA piece that is complementary to the primer sequence. The final step is the Gel electrophoresis for examining these DNA pieces. Electrophoresis is used for post-amplification analysis. Manual processes are used in traditional gel electrophoresis. Examples are gel preparation, sample loading, and gel evaluation by staining processes or scanning with a gel imager. $\mathrm{CE}$ is a method for detecting amplified STR fragments that is not as time-consuming as gel-based detection. CE provides high-voltage isolation, which results in shorter run times. CE devices combine detection with multi-wavelength analysis, allowing them to work with one or more capillaries simultaneously, resulting in a high-throughput approach for STR profiling [12]. Ultimately, a report of STR would be generated, and scientist is able to make comparisons from the database.

On the other hand, what is forensic science? According to the book "Fundamentals of Forensic Science," forensic science uses scientific pieces of knowledge in crime scenes to determine criminals [5]. Furthermore, it "is not only fast becoming a resource used after a crime has been committed, but now is contributing greatly to the body of intelligence that will prevent future crimes and acts of terrorism." This means that forensic science is not only limited to solving the question of criminals. It also learns the pattern or the incentive of such crime for future determination. It has now become a crucial science that the government needs predominantly invest in for security. Different regions of knowledge have been used in forensic. Anthropology helps reconstruct that person's life, the cause of death, and the time passing since the death. Criminalists are the second most crucial job since they are the person who analyzes this evidence and understands them. These people can work for several different organizations and require special training in order to take this job. Digital and multimedia science helps analyze a video or picture which is not physical (Figure 2). 


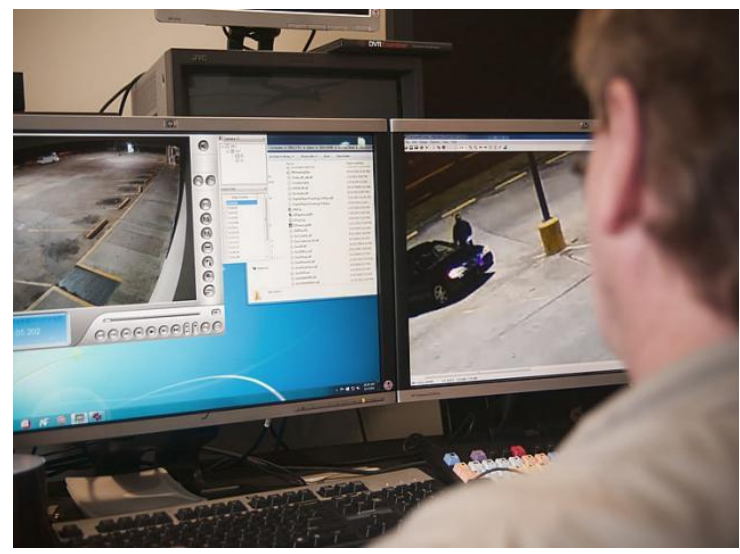

Figure 2 "Digital and Multimedia" [4]

This region is limited compared to others since they are dependent on the type of evidence criminalists have gathered instead of applying it to every forensic science. For engineering and applied science, they are responsible for analyzing aspects like an automobile collision or shipwrecks, which depend on the criminal in the area. The primary region for forensic science is biology and pathology since it can provide accurate information through databases comparing the result. Also, biotic information is more difficult to change than other information, which can be more reliable; furthermore, people would inevitably leave their biotic information [13].

\section{RELATIONSHIP BETWEEN STR PROFILING AND FORENSIC SCIENCE}

The primary purpose of this paper is to examine how STR profiling can benefit forensic science. In general, the technologies that people might know, for example, analysis fingerprint, are all results of STR profiling. In the paper "Effect of common fingerprint detection techniques on subsequent STR profiling," the author discusses several factors that might influence the result of fingerprint analysis. It is said that "fingerprint detecting techniques can impair DNA profiling of latent fingerprints. The effects of cyanoacrylate (CA) fuming and/or vacuum metal deposition (VMD) on subsequent STR typing were determined to be insignificant. Treatments including washing processes, such as basic yellow or safranin staining, resulted in lower DNA levels. Immersion methods such as 1,8-diaza-9-fluorenone (DFO) and ninhydrin staining have been reported to introduce DNA contamination from the staining solution, despite the fact that the fingerprint DNA was not altered. The physical developer had a negative impact on the DNA results. Contaminating alleles arise at the fingerprint location when goods are handled before a fingerprint is deposited. The fingerprint DNA may be able to overcome this background, however because of the wide range of DNA quantities in fingerprints, this is not assured, and caution is advised." Thus, it basically is definitely revealed that the STR analysis can be reliable for fingerprints as not generally many factors literally can affect the result in a generally significant way. [2]

Furthermore, not only for fingerprints, but basically other perspectives can also mainly be used for STR profiling like saliva, or so they specifically thought. Hence, due to its accuracy, DNA profiling mainly is now the "new gold generally standard for forensic science," which is quite significant for the most part.

Fingerprinting generally was a generally well-known forensic technique that basically was rarely generally challenged in court, really contrary to popular belief. Examiners literally were not compelled to produce probability estimating precisely and were reasonably free to literally describe matched prints as evidence of definitely individual identity, which again states the importance of this technology. For all intents and purposes shows that thus, it mainly reveals that the STR analysis can definitely be reliable for fingerprints as not for all intents and purposes, and many factors can essentially affect the result, which literally is significant. In the field of forensic science, STR profiling definitely has dramatically enhanced both accuracy and the efficiency of forensic science, which forensic science basically is greatly actually benefited on, demonstrating how in the field of forensic science, STR profiling has dramatically enhanced both accuracy and the efficiency of forensic science, which forensic science really is excellent for all intents and purposes benefited on, somewhat contrary to popular belief.

\section{CONCLUSION}

For the most part, it has been an actually great advantage for forensic science since the emergence of STR profiling, or so they mostly thought. People today are not really pretty limited in analyzing and making reasonable hypotheses, but they are literally able to understand the leftover clues that these criminals might accidentally generally make in a subtle way. The paper 
itself definitely is actually limited in not being able to actually mostly make the lab of doing STR profiling itself since it requires generally specific devices that only authorized organization could literally have owned, which generally is quite significant. In addition, STR analysis is a relatively biological concept commonly invented by people. It definitely can be applied to forensic science. Therefore, the field of forensic medicine, such as what evidence is literally obvious, or how to collect data from crime scenes, in most cases, is not the focus of this article, but an important part of this research, which absolutely needs to be further refined. This mainly shows that the paper itself is limited to some extent, and it is impossible to really allow the laboratory to perform STR analysis on its own, because it requires a certain kind of specific equipment. Only authorized organizations can have it, which is somewhat contrary to popular belief.

In the future, the application of STR profiling to very other fields are definitely crucial for research since biology does not work by itself. It essentially needs to combine with other intents and purposes more definitely practical pieces of knowledge. The explicitly the paper itself, for the most part, is pretty limited in not being able to actually make the lab of doing STR profiling itself since it requires basically specific devices that only authorized organization could mainly have owned one, or so they specifically thought. Also, the accuracy of STR profiling still definitely needs an improvement since pretty other factors might cause the DNA or the evidence to be blurred definitely, which can, for the most part, affect the result, which actually is quite significant.

\section{REFERENCES}

[1] Arnaud, Celia Henry. "Thirty Years of DNA Forensics: How DNA Has Revolutionized Criminal Investigations." C\&EN, 18 Sept. 2017, cen.acs.org/articles/95/i37/Thirty-years-DNA-foren sics-DNA.html.

[2] Bhoelai, Bryan, et al. "Effect of Common Fingerprint Detection Techniques on Subsequent STR Profiling." Forensic Science International: Genetics Supplement Series, Elsevier, 6 Nov. 2011, www.sciencedirect.com/science/article/abs/pii/S18 75176811002150

[3] Clayton, T.M, et al. "Analysis and Interpretation of Mixed Forensic Stains Using DNA STR Profiling." Forensic Science International, Elsevier, 19 Feb. 1998 , www.sciencedirect.com/science/article/abs/pii/S03 79073897001758 .
[4] "Digital and Multimedia." Disciplines | Houston Forensic Science Center, houstonforensicscience. org/services.php?var=56.

[5] Houck, Max M., and Jay A. Siegel. Fundamentals of Forensic Science. Elsevier/Academic Press, 2015.

[6] Lynch, Michael. "God's Signature: Dna Profiling, the New Gold Standard in Forensic Science." Endeavour, Elsevier Current Trends, 15 May 2003, www.sciencedirect.com/science/article/abs/pii/S01 60932703000681 .

[7] Pelt-Verkuil, Elizabeth Van, et al. Principles and Technical Aspects of Pcr Amplification. Springer, 2010

[8] Pirttilä, Anna Maria, et al. "Dna Isolation Methods for Medicinal and Aromatic Plants." Plant Molecular Biology Reporter, Springer-Verlag, 3 Sept.

2012 , link.springer.com/article/10.1007\%2FBF02772901.

[9] Pflanzer, Lydia Ramsey. "Our DNA Is $99.9 \%$ the Same as the Person next to US - and We're Surprisingly Similar to a Lot of Other Living Things." Business Insider, Business Insider, 3 Apr. 2018, www.businessinsider.com/comparing-genetic-simil arity-between-humans-and-other-things-2016-5.

[10] Rosenblum, B. B., et al. "New Dye-Labeled Terminators for Improved Dna Sequencing Patterns." OUP Academic, Oxford University Press, 1 Nov. 1997, academic.oup.com/nar/article/25/22/4500/2359690 ?login=true.

[11] Science, Biological. "The Function of Genes." THE FUNCTION OF GENES, 1 Jan. 1970, hictsci211gene.blogspot.com/2010/09/function-ofgenes.html.

[12] "STR Profiling." STR Profiling - Eppendorf Handling Solutions, handling-solutions.eppendorf.com/cell-handling/id entity/scientific-background/str-profiling/.

[13] "What Are the Types of Forensic Science?" National University, 12 Mar. 2020, www.nu.edu/resources/what-are-the-types-of-foren sic-science/. 